\title{
Management of intramedullary astrocytomas
}

\author{
Daniel Serban, Florin Exergian, R.M. Gorgan \\ "Bagdasar-Arseni" Clinical Emergency Hospital, Neurosurgery I, Spinal Surgery, \\ Bucharest
}

\begin{abstract}
Primitive IMT represent $8-10 \%$ of all primary tumors of the spinal cord. Only 2$4 \%$ of all CNS tumors in adults are IMT. Adult astrocytomas are $25-35 \%$ of total IMT (1).

We prospectively analyzed clinical, imaging and pathological data from all consecutive patients operated for intramedullary tumors in our department (Neurosurgery I Clinic, Ward II) between January 2003 and August 2009 (80 months). All surgical interventions were performed by the same surgical team. We emphasized the technical difficulties raised by ablation of IMT depending on the type of the tumor and postoperative neurological outcome.
\end{abstract}

Key words: intramedullary astrocytomas, surgery, postoperative neurological outcome.

Abreviations: CNS - central nervous system, intramedullary tumors - IMT, spinal cord - SC.

\section{General data}

Primitive IMT represent $8-10 \%$ of all primary tumors of the spinal cord. Only 2$4 \%$ of all CNS tumors in adults are IMT. SC tumors are much less common than intracranial tumors with an overall prevalence of approximately an intramedullary tumor for four intracranial tumors, with variations depending on the type of tumor. For example, the location intracranial/spinal for astrocytomas is approximately 10/1, while the same ratio for ependymomas varies from $3 / 1$ to $20 / 1$ depending on the histological type of ependymoma. In particular, mixo-papillary ependymomas are found more frequently in the SC (1).

\section{Material and methods}

Patients enrolled in the study were hospitalized and operated in the period 2003-2009 in Neurosurgery I Clinic, Ward II, "Bagdasar-Arseni" Clinical Emergency Hospital for IMT in various locations. 59 patients were included in the study, age between 15 and 70 years, $40 \%$ female sex ratio $=1.5$. 62 surgeries were performed. All patients were operated on by the same surgical team, same main operator.

\section{Result distribution}

In the series we have studied the distribution by frequency of pathological diagnoses found is the following:

- first place is occupied by ependymomas, which account for about half of all pathological types - 29 cases $(46.77 \%)$

- on the second place are astrocytomas 15 cases ( $24.19 \%)$

- third place hemangioblastomas 5 cases $(8.06 \%)$

- the fourth place two lipomas and a 
fibrolipoma summing 3 cases $(4.83 \%)$

- on the fifth place are cavernomas, meduloblastomas, gangliogliomas, schwanomas by 2 cases $=3.22 \%$ each, summimg 8 cases $=12.88 \%$

- the sixth place epidermoid cyst and a metastasis 1 case $=1.61 \%$ each, totaling 2 cases $=3.22 \%$

Note that the first three histological types, ependymomas, astrocytomas, hemangioblastomas adds up to approx. $80 \%$ of all IMT in adults. All other seven histological types account for the remaining $20 \%$

Analysis of tumor histology for astrocytomas revealed predominant WHO grades I and II - 11 cases, compared with grades III and IV - 4 cases. The most common subtype was fibrillary astrocytoma, 6 cases.

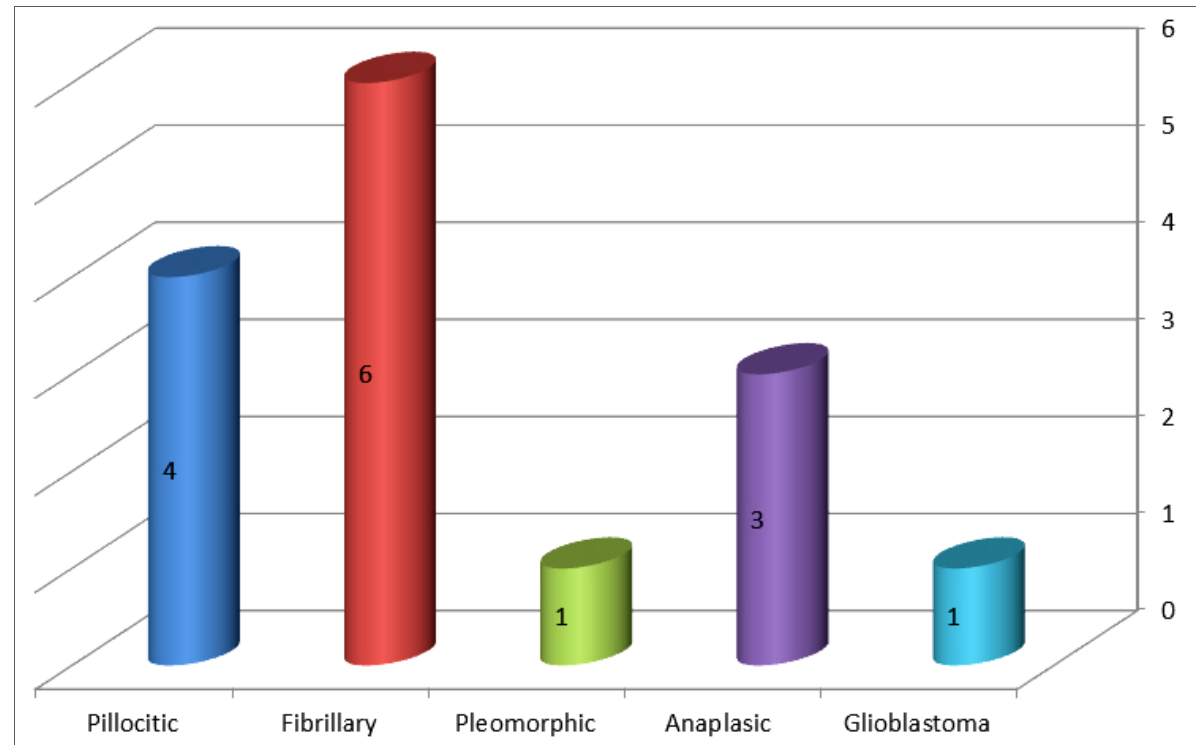

Figure 1 Pathological types of astrocytomas

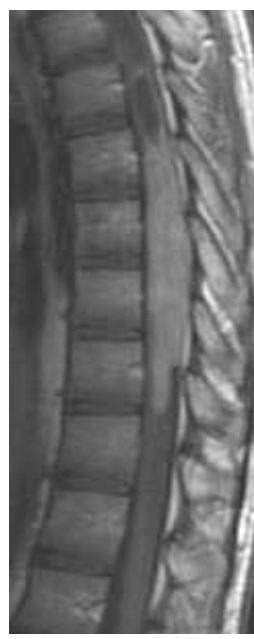

A

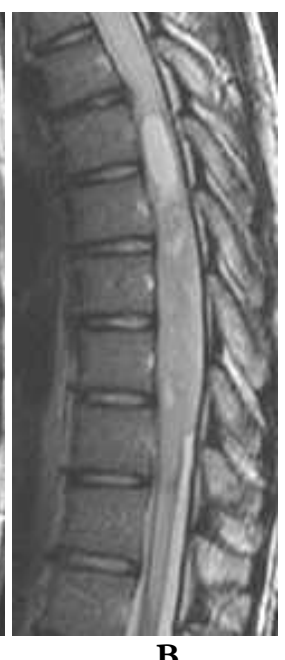

B

Figure 2 Thoracic pilocitic astrocytoma, preoperative

A - T1 image, dye examination B - T2 image

Preoperative diagnosis was made by MRI in all cases, native examination and T1-dye examination.

Location:

- 2 cervical astrocytomas - 13.33\% (of total astrocytoma localization)

- 1 cervico-thoracic astrocytoma - 6.66\%

- 12 thoracic astrocytomas $-80.00 \%$

Most frequent location is the thoracic spine region - $80 \%$. Astrocytomas occupied between 2 and 4 vertebral segments, with an average of 2.86 vertebral levels. 


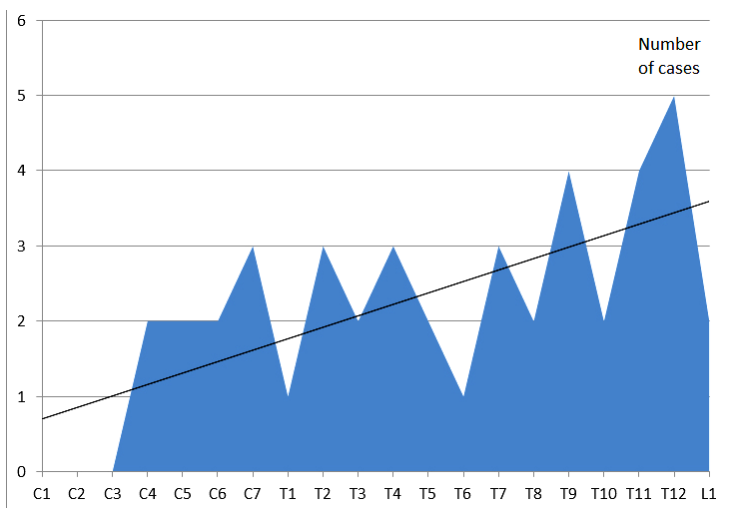

Figure 3 Astrocytomas location relative to the vertebral segments (abscissa - C1 to L1 vertebral segments, the ordered number of cases each vertebral level)

\section{Surgery}

Anesthesia, patient positioning and wound opening, laminoplasty or laminectomy, opening and suspension of dura mater and arachnoid and wound closure are similar to surgery for any IMT.

Myelotomy is more difficult for astrocytomas compared to the same maneuver for ependymomas (2). These problems are caused by the fact that astrocytomas are not developed in the midline, are IMT with asymmetric development. In addition the SC may be "distorted", anatomical landmarks being no longer valid. Whenever possible, it is recommended myelotomy in the posterior midline. Finding solutions for posterior midline are coagulation and cutting tortuous veins usually found on the posterior surface of the SC and subsequent search for the posterior midline in the area where SC have anatomic appearance above and below the area of astrocytoma development.

Another solution is mielotomy in the dorsal root entry zone (DREZ) where the extension of the tumor is greater and neurological deficits are higher. As ultimate solution is used the myelotomy in the area where astrocytoma is closest to the posterior surface of the SC, the aim being to harm as little as possible the medullary tissue.

Astrocytoma biopsy to determine the degree of malignancy is the next step, which may be decisive for the further conduct of the surgeon. If biopsy results revealed low-grade astrocytoma, we always tempted total or almost total ablation of astrocytoma. Although pathological results showed high-grade astrocytoma we was looking for an eventual cleavage plane and tried subtotal or nearly total ablation of astrocytoma. The same attitude was adopted in situations where polar cysts were encountered. We started to search for the cleavage plane between the tumor and normal medullary tissue, near the cysts. Always be dissected with extreme caution, lateral sides of astrocytomas, which may come in contact with cortico-spinal tracts and the front of astrocytomas, that sometimes "stuck" anterior spinal artery. Cortico-spinal tracts damage and/or anterior spinal artery lesions causes significant postoperative neurological deficits, most often irreversible $(3,4,5)$.

Tumor ablation technique used was intratumoral partial ablation, emphasizing the "step by step" plan and finding the cleavage plane fragment by fragment. The internal pressure relief stitch was used in 2 cases when the astrocytoma extended for 4 vertebral levels. Whatever type of tumor ablation, hemostasis was achieved by mild pressure, washing the wound with cold saline and wallpapering outstanding intramedullary cavity with Surgicel.

Type of tumor ablation

Total ablation was possible in 2 cases (13.33\%), a T2-T4 thoracic pilocytic astrocytoma and a fibrillary astrocytoma 
T9-T10, situations in which was possible to found a cleavage plane.

Nearly total ablation was performed in one case $(6.66 \%)$, a pilocytic astrocytoma T11- L1.

The most common was subtotal ablation in 10 cases $(66.66 \%)$ being imposed by the infiltrative nature of tumor formation (glioblastoma one case, 3 cases of anaplastic astrocytoma, diffuse infiltrative fibrillary astrocytoma grade II / III WHO one case) and unable to find a plane of cleavage between the IMT and normal medullary tissue in the other cases (2).

Biopsy was chosen as a temporary solution in two cases (13.33\%), in which intra-operative cardiovascular disorders were recorded, the primary surgeon's decision was to minimize the duration of the intervention. Pathological results were pilocytic astrocytoma in one case and fibrillary astrocytoma in the other. A second intervention was scheduled within the first 14 days postoperatively performing the almost total ablation in the first case and the subtotal ablation in the second case.

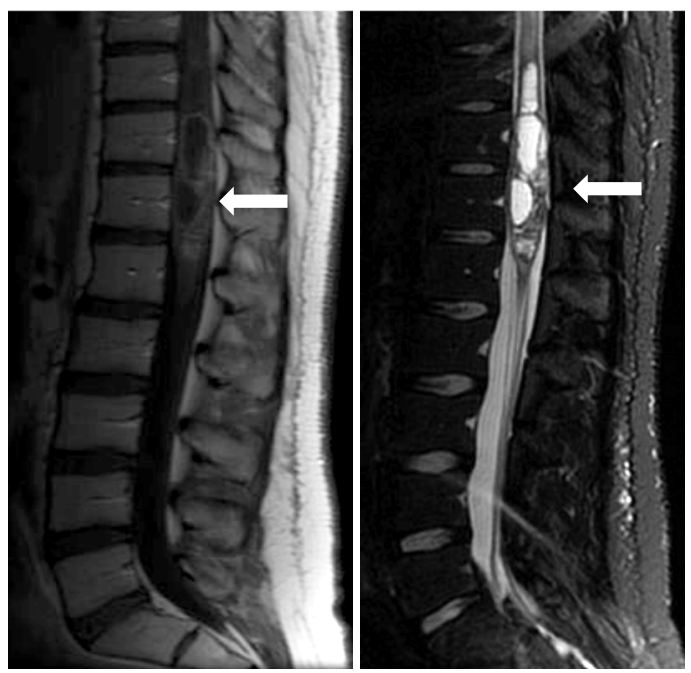

Figure 4 T1, sagital; T2, sagital Pilocitic astrocytoma
Tumoral ablation

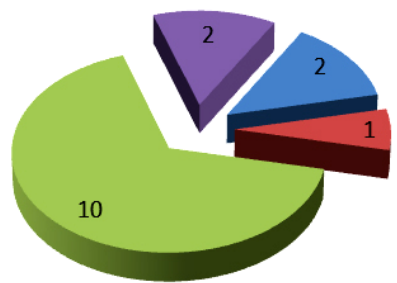

n total

quasitotal

nubtotal

घ biopsy

Figure 6 Types of tumor ablation

\section{Intraoperative complications}

Intraoperative bleeding was not significant except for one case; the thoracic astrocytoma was extended on 4 levels. In the two cases described above, in which biopsy was performed only at the first surgery, complications are caused by cardiovascular pathology that patients already had and were not related to location or type of excision of IMT.

Postoperative complications

No significant postoperative complications were recorded.

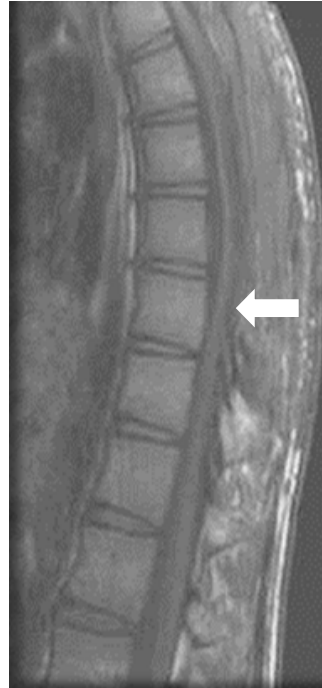

A

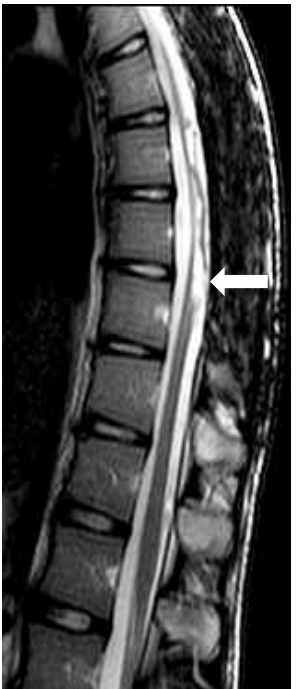

B
Figure 5 Thoracic pilocitic astrocytoma, six months postoperative

A - T1 dye examination, image B - T2 image 


\section{Discussion}

\section{Postoperative neurological evolution}

There were no significant neurologic deficits. Additional motor deficit was averaging 1.2 points on ASIA scale and sensitivity disorders, except deep sensibility disorders, not widened. Favorable evolution from both surgical and neurological point of view is related to the location of IMT and the type of ablation. The tumors in low thoracic spinal cord had the lower critical risk (in our series there were no astrocytomas located above C4). The type of tumor resection is also extremely important: there were only 2 cases with total resection and 2 cases with subtotal resected tumors.

In other words, if the IMT location is "high" and/or tumor ablation attempt more radical (total or almost total), the vital and neurological risks and complications are higher $(6,7,8,9)$.

Postoperatively there has been no significant neurological worsening, no death registered.

Explanations are:

- Type of ablation that was only in 2 cases total and in one case quasi total determined by the presence of cleavage plane;

- Predominantly astrocytomas location in thoracic levels, 12 cases, only 2 locations cervical and 1 cervico-thoracic junction 1;

- Surgery less "aggressive" if the pathological results were high-grade astrocytomas.

Type of tumor ablation should be adapted to histological subtype of astrocytoma and especially must take into account the existence or absence of a cleavage plane $(8,9)$. Brotchi (2) in an article published in 1997 emphasizes that any surgical attitude addopted, if the astrocytoma is grade III or IV, long-term results are similar. In these cases the surgeon's duty is to obtain a correct pathological result, to achieve decompression of the SC without affecting medullary tissue, without postoperative neurological deterioration.

\section{Adjuvant treatment}

\section{Radiation}

Radiation therapy has no indication for low-grade astrocytomas. All authors (2, 3, 4, $5,6)$ agree that in pilocytic astrocytoma WHO grade I postoperative radiotherapy should not be done, just followed up the evolution by clinical and imaging surveillance. In these cases the neurological evolution, the risk of relapse and duration of postoperative survival are directly related to the type of tumor ablation, total or that sometimes occur after radiation: myelitis postirradiation and bone-marrow suppression.

For high-grade gliomas, WHO grade III and IV was made postoperative radiotherapy, fractionated doses of 50-54 Gy in 24 sessions.

For grade II gliomas WHO histological with features of malignancy and recurrent gliomas grade II radiotherapy was made with doses of 40-46 Gy, fractionated in 20 sessions (10).

Postoperative radiotherapy was done in patients with WHO grade III anaplastic astrocytomas (3 cases), glioblastoma WHO grade IV (1 case) and in WHO grade II astrocytomas with anaplastic histological elements (1 case) in which we could not perform complete ablation.

\section{Chemotherapy}

It was recommended as adjuvant therapy in the pediatric population because of disastrous side effects that radiation has on children. For adults was indicated in cases 
where astrocytomas are $\mathrm{WHO}$ grade III and IV, IMT resection was not complete, did not respond to radiotherapy and tumor recurrences were recorded or "tumoral growth" continues. Etoposide was used initially, then Temozolamide. Temozolamide administered in doses of 150-200 mg./m2/day for 5 days a week for 4 weeks $=$ a cycle (11).

In the present series we did not have patients with postoperative chemotherapy.

\section{Conclusions}

- Astrocytomas grade I could be completely or partially ablated. Total or almost total ablation is due to the cleavage plane between IMT and normal medullary tissue. In low-grade astrocytomas, where there plane cleavage is present, total or almost total ablation is the goal.

- Astrocytomas grade III and IV and part of grade II astrocytomas (with anaplastic cells elements) were subtotally ablated because of their infiltrative nature.

- There were no major intraoperative complications, postoperative immediately and/or delayed.

- All cases of grade III and IV astrocytomas have clear indication for postoperative radiotherapy.

\section{References}

1. Dolecek TA, Propp JM, Stroup NE, Kruchko C. CBTRUS Statistical Report: Primary brain and central nervous system tumors diagnosed in the United States in 2005-2009. Neuro-Oncol.2012;14 ( 5): v1-v49

2. Brotchi J. Intramedullary spinal cord astrocytomas: diagnosis and treatment. Crit Rev Neurosurg. 1997;7:83-88.

3. Cristante LHH. Surgical management of intramedullary spinal cord tumors: functional outcome and sources of $\mathrm{m} 8$. Grimm S., Chamberlain M.C. Adult primary spinal cord tumors. Expert Rev. Neurother. 2009; 9(10) : 1487 - 95.

4. Epstein F.Surgical management of intramedullary spinal cord tumors : functional outcome and sources of morbidity (comment). Neurosurgery.1994; 35: 76

5. Chamberlain MC, Tredway TL. Adult primary intradural spinal cord tumors: a review. Curr Neurol Neurosci Rep. 2011; 11(3):320-8.

6. Garcés-Ambrossi GL, McGirt MJ, Mehta VA, Sciubba DM, Witham TF, Bydon A, et al. Factors associated with progression - free survival and longterm neurological outcome after resection of intramedullary spinal cord tumors: analysis of 101 consecutive cases. Clinical article. J Neurosurg Spine. 2009; 11:591-599

7. Harrop JS, Ganju A, Groff M, Bilsky M. Primary intramedullary tumors of the spinal cord, Spine. 2009; 34,(22S), S69-77

8. Kim MS, Chung CK, Choe G, Kim IH, Kim HJ. Intramedullary spinal cord astrocytoma in adults: postoperative outcome. Journal of NeuroOncology.2001; 52: 85-94

9. V Benes V 3rd, Barsa P, Benes V Jr, Suchomel P. Prognostic factors in intramedullary astrocytomas: a literature review. Eur Spine J .2009; 18:1397-1422

10. Isaacson Steven R. Radiation therapy and the management of intramedullary spinal cord tumors. Journal of Neuro-Oncology.2000, 47: 231-238

11. Chamberlain M.C. Temozolomide for recurrent low-grade spinal cord gliomas in adults. Cancer. 2008;113(5):1019-1024. 